\title{
Method of Boundary Layer Function to Solve the Boundary Value Problem for a Singularly Perturbed Differential Equation of the Order Two with a Turning Point
}

\author{
Keldibay Alymkulov \\ Institute of Fundamental and Applied Researches of Osh State University, Osh c., 723504, Kyrgyzstan \\ *Corresponding Author: keldibay@mail.ru
}

Copyright (C 2014 Horizon Research Publishing All rights reserved.

\begin{abstract}
The solution of the boundary value problem is constructed singularly perturbed differential equation of the order two with the turning point by method of boundary layer function.
\end{abstract}

Keywords Singularly Perturbed Differential Equation, Turning Point, Method of Boundary Layer Functions or Method of Composite Expansion, Method of Structurally Matching, Contraction Mapping Principle

Mathematics Subject Classification (2010): 34E05, 34E10

\section{Introduction}

Apparently, for the first a uniformly asymptotic expansion of a solution of a singularly perturbed linear equations with small parameter in higher derivatives(equations of Prandtl-Tikhonov type, shortly P-T type) was constructed G.E.Latta in 1951 [1] and E.Bromberg in 1956 [2] by method of composite expansions. I note that this method of composite expansion in soviet mathematicians is called method of boundary layer functions (briefly MBLF).

But a uniformly asymptotic expansion of solutions of nonlinear equations type P-T received by A.B. Vasilievain1960 only [3]-[5] by the method of matching, after by W.Wasow and Y. Sibuya in 1963 [3].

The MBLF for linear singularly perturbed partial differential equations of type P-T was developed by M.I.Vishik and L.A. Lusternik in 1957 [6] and for nonlinear differential equations ( more exactly: for the singulary perturbed integro-differential equations) by M.I. Imanaliev 1964 [7], after by R.E.O’MalleyиF.Hoppensteadt 1971 [3] . More surveys of related material are contained in [3], [8][11].

The MBLF usually is used for the construction of asymptotic solution of the singularly perturbed equations in the case of exponential asymptotical stability of the equation in the fast variable, i.e. under the A.N. Tikhonov theorem's conditions.

Also, for the construction of asymptotic solutions of the singularly perturbed equations the method of matching outer and inner solution is applied [10-16] and the rule of matching of the outer and inner solution was given by Van Dyke. I note that the developing of the method matching was given in [17]-[20].

Here it is proved the possibility of using of the MBLF for construction of the asymptotic of the boundary value problem for equations with the turning point, where the Tihonov's condition is broken.

\section{Statement of the Problem}

The following problem is considered

$$
\begin{gathered}
\varepsilon y^{\prime \prime}(x)+x y^{\prime}(x)-q(x) y(x)=f(x) \\
y(0)=0, y(1)=y^{0}
\end{gathered}
$$

where $0<\varepsilon<<1$-small parameter, $x \in[0,1], y^{0}$ - is given constant. The following condition $U: q(x), f(x) \in C^{\omega}[0,1]$ (that is class analytical functions) is imposed on the known functions. It follows that

$$
\begin{aligned}
& q(x)=\sum_{k=0}^{\infty} q_{k} x^{k}, f(x)=\sum_{0}^{\infty} f_{k} x^{k}, \\
& f_{k}=f^{(k)}(0) / k !, \quad q_{k}=q^{(k)}(0) / k !
\end{aligned}
$$

Here we will consider the case $q_{0}=q(0)=1$ for simplicity.

I note that the solution of the problem (1)-(2) was constructed earlier by method of boundary layer functions with the application of differential inequality in [20] under the condition $q(x)>1$. Further the asymptotic of the solution of the problem (1)-(2) was constructed by the 
method of structural matching [17-18] in [19].

\section{Construct of the Solution of the Problem by the Method of the Boundary Layer Functions}

The solution of the unperturbed equation (1) $(\varepsilon=0)$

$$
M y_{0}:=x y_{0}^{\prime}(x)-q(x) y(x)=f(x), y(1)=y^{(0)},
$$

is represented as follows

$$
\begin{aligned}
& y_{0}(x)=x p(x)\left[y^{(0)}+\int_{1}^{x} r(s) d s / s^{2}\right], \\
& r(x)=p^{-1}(x) f(x), \\
& p(x)=\exp \left\{\int_{1}^{x}[(q(s)-1) / s] d s\right\} .
\end{aligned}
$$

By distinguishing the main part of the integral in the sense of Hadamar [21] in (4) we may represent this one to next form

$$
y_{0}(x)=a(x)+r[f] x p(x) \ln x,
$$

where

$$
\begin{aligned}
& a(x)=x p(x)\left[z(1)+\int_{1}^{x}\left(r(s)-r_{0}-r_{1} s\right) d s / s^{2}\right], \\
& \left.r_{0}=p^{-1}(0) f(0), r_{1}=r[f]=\left[p^{-1}(x) f(x)\right)^{\prime}\right]\left.\right|_{x=0} .
\end{aligned}
$$

The formula(5) implies

Lemma 1. The problem

$$
M y_{0}(x)=f(x)-r[f] x p(x), y(1)=y^{0},
$$

have a unique solution in $C^{(\infty)}[0,1]$ and $y_{0}(x)=a(x)$, where $a(x)$ is defined by (6).

We will represent the solution of the problem (1)-(2) in the following form

$$
\begin{aligned}
& y(x)=y_{0}(x)+\pi_{0}(t)+\mu\left(y_{1}(x)+\pi_{1}(t)\right)+ \\
& +\mu^{2}\left(y_{2}(x)+\pi_{2}(t)\right)+\ldots+\mu^{n}\left(y_{n}(x)+\pi_{n}(t)\right)+\ldots
\end{aligned}
$$

where $\quad t=x / \mu, \varepsilon=\mu^{2}, \pi_{k}(t) \in C^{(\infty)}[0, \tilde{\mu}), \tilde{\mu}=\mu^{-1}$ $y_{k}(x) \in C^{(\infty)}[0,1]$. We denote, that the function $\pi_{k}(t)=\pi_{k}(t, \mu)$, i.e. $\pi_{k}(t)$ depends on $\mu$, but we will not write it for brevity.

We will take initial data for functions $y_{k}(x) \in C^{\infty}[0,1]$ and $\pi_{k}(t)$ in the form

$$
\begin{aligned}
& y_{0}(1)=y^{(0)}, y_{k+1}(1)=0 ; \pi_{k}(0)=-y_{k}(0), \\
& \pi_{k}(\tilde{\mu})=0(k=0,1,2, \ldots) .
\end{aligned}
$$

By inserting series (7) to (8) to determine functions $y_{k}(x)$ and $\pi_{k}(t)$ we receive the following equations

$$
\begin{gathered}
M y_{0}(x)=f(x), y_{0}(1)=y^{0}, \\
L_{\mu} \pi_{0}(t):=\pi_{0}^{\prime \prime}(t)+t \pi_{0}^{\prime}(t)-q(\mu t) \pi_{0}(t)=0 \\
\pi_{0}(0)=-y_{0}(0), \pi_{0}(\tilde{\mu})=0 \\
M y_{1}(x)=0, y_{1}(1)=0 \Rightarrow y_{1}(x)=0, \\
L_{\mu} \pi_{1}(t)=0, \pi_{1}(0)=\pi_{1}(\tilde{\mu})=0 \\
M y_{2}(x)=-y_{0}(x):=f_{2}(x), y_{2}(1)=0, \\
L_{\mu} \pi_{2}(t)=0, \pi_{2}(0)=-y_{2}(0), \pi_{2}(\tilde{\mu})=0 \\
M y_{2 n-1}(x)=0, y_{2 n-1}(0)=0 \Rightarrow y_{2 n-1}(x)=0,(8.2 \mathrm{n}-1) \\
L_{\mu} \pi_{2 n-1}(t)=0, \pi_{2 n-1}(0)=-y_{2 n-1}, \pi_{2 n-1}(\tilde{\mu})=0(9.2 \mathrm{n}-1) \\
M y_{2 n}(x)=-y_{2 n-2} "(x), y_{2 n}(1)=0 \\
L_{\mu} \pi_{2 n}(t)=0, \pi_{2 n}(0)=-y_{2 n}(0), \pi_{2 n}(\tilde{\mu})=0,(9.2 \mathrm{n})
\end{gathered}
$$

Lemma1 implies, that functions $y_{k}(x)$ can't be defined from in $C^{\infty}[0,1]$ with arbitrary functions $f(x)$. Therefore we must change right hand parts equations $(8.2 \mathrm{n})$ and respectively right hand part equations $(9.2 \mathrm{n})$. We will change them as follows

$$
\begin{gathered}
M y_{0}(x)=f(x)-r[f] x p(x), y_{0}(x)=y^{0}, \\
M y_{2 n}=-y_{2 n-2} "(x)+r\left[y_{2 n-2} "\right] \cdot x p(x), \\
y_{2 n}(1)=0(n=1,2, \ldots) \\
L_{\mu} \pi_{0}(t):=\pi_{0}^{\prime \prime}(t)+t \pi_{0}^{\prime}(t)-q(\mu t) \pi_{0}(t)= \\
=r[f] \mu t p(\mu t), \pi_{0}(0)=-y_{0}(0), \pi_{0}(\tilde{\mu})=0, \\
L_{\mu} \pi_{2 n}(t)=-r\left[y_{2 n-2} "\right] \cdot \mu t p(\mu t), \\
\pi_{2 n}(0)=-y_{2 n}(0), \pi_{2 n}(\tilde{\mu})=0
\end{gathered}
$$

Now all functions $y_{k}(x)(k=0,1,2 \ldots)$ will be defined in $C^{\infty}[0,1]$.

To define the functions $\pi_{k}(t) \quad(\mathrm{k}=0,1,2, \ldots)$ we need the following

Lemma 2.The homogeneous equation

$$
L_{0} \xi(t)=\xi^{\prime \prime}(t)+t \xi(t)-\xi(t)=0
$$

has two linearly independent solutions :

$$
X_{1}(t):=X(t)=t \int_{t}^{1 / \mu} e^{-s^{2} / 2} s^{-2} d s, X_{2}(t)=t, X(0)=1
$$

Integrating $X(t)$ by parts integral of we have got 


$$
\begin{aligned}
& X(t)=t^{-2}\left[1-3 t^{-2}+3.5 t^{-4}-\ldots+\right. \\
& \left.+(-1)^{n} 1 \cdot 3 \cdot 5 \cdots(2 n-1) t^{-2 n-1}\right] e^{-t^{2} / 2}- \\
& -t \mu^{3}\left[1-3 \mu^{-2}+3.5 \mu^{-4}-\ldots+\right. \\
& \left.(-1)^{n} 1 \cdot 3 \cdot 5 \cdots(2 n-1) \mu^{-2 n-1}\right] e^{-1 / 2 \mu^{2}}+ \\
& +(-1)^{n} 1 \cdot 3 \cdot 5 \cdots(2 n-1)(2 n+1) \int_{t}^{\tilde{\mu}} s^{-2 n-2} e^{-s^{2} / 2} d s .
\end{aligned}
$$

From this formula and previous equality we have got:

$$
\begin{aligned}
& \text { a) } \begin{array}{l}
0 \leq X(t) \leq e^{-t^{2} / 2} \leq 1 ; 0 \leq X(t) \leq t^{-2} e^{-t^{2} / 2} \\
X^{\prime}(t) \leq 0, t>0
\end{array} \\
& \text { b) } 0 \leq X(t) \leq t^{-2} e^{-t^{2} / 2}\left(1-3 t^{-2}+3 \cdot 5 t^{-4}\right),(\mathrm{t}>0) . \\
& \text { c) } X(t)=\left\{\begin{array}{l}
1+0(t), t \rightarrow 0, \\
e^{-t^{2} / 2} t^{-2}\left(1-3 t^{-2}+0\left(t^{-4}\right)\right), t \rightarrow \infty
\end{array}\right.
\end{aligned}
$$

Lemma 3. The function of Green of the boundary value problem $\xi(0)=\xi(\tilde{\mu})=0$ for the equation (11) is defined the following formula

$$
G(t, s)=\left\{\begin{array}{l}
-t X(s), 0 \leq t \leq s \\
-X(t) s, s \leq t \leq \tilde{\mu} .
\end{array}\right.
$$

Lemma 4. The solution of the problem

$$
L \xi(t)=f(t), \quad \xi(0)=\xi(\tilde{\mu})=0
$$

where $f(t) \in C[0, \tilde{\mu}]$ is represented in the form

$$
\begin{aligned}
& \xi(t)=\int_{0}^{\tilde{\mu}} K(t, s) f(s) d s= \\
& =-t \int_{t}^{\tilde{\mu}} \tau^{-2} e^{-\tau^{2} / 2} \int_{0}^{\tau} s e^{s^{2} / 2} f(s) d s d \tau:=K[f]
\end{aligned}
$$

where $K(t, s)=e^{s^{2} / 2} G(t, s)$.

Also if $|f(t)| \leq m, t \in[1, \tilde{\mu}]$, then $|\xi(t)| \leq m$.

Proof. Using $K(t, s)$ we have

$$
\begin{aligned}
& \xi(t)=-\int_{0}^{t} X(t) s e^{s^{2} / 2} f(s) d s- \\
& -t \int_{t}^{\tilde{\mu}} \int_{s}^{\tilde{\mu}} \tau^{-2} e^{-\tau^{2} / 2} d \tau s e^{s^{2} / 2} f(s) d s
\end{aligned}
$$

After changing the order of integrating we have got

$$
\begin{aligned}
& \int_{t}^{\tilde{\mu}} \int_{s}^{\tilde{\mu}} \tau^{-2} e^{-\tau^{2} / 2} d \tau s e^{s^{2} / 2} f(s) d s= \\
& \int_{t}^{\tilde{\mu}} \int_{t}^{\tau} s e^{s^{2} / 2} f(s) d s \tau^{-2} e^{-\tau^{2} / 2} d \tau= \\
& =\int_{t}^{\tilde{\mu}} \int_{0}^{\tau} s e^{s^{2} / 2} f(s) d s \tau^{-2} e^{-\tau^{2} / 2} d \tau- \\
& -t^{-1} \int_{0}^{t} X(t) e^{s^{2} / 2} f(s) d s
\end{aligned}
$$

Evaluation of the $\xi(t)$ is evident.

Lemma 5 . The boundary problem

$$
\begin{aligned}
& \xi^{\prime \prime}(t)+t \xi^{\prime}(t)-q(\mu t) \xi=f(t), \\
& \xi(0)=\xi(\tilde{\mu})=0
\end{aligned}
$$

where $|f(t)| \leq m$, have unique bounded

solution on the interval $[0, \tilde{\mu}]$.

Proof. The problem (16) equivalent to the following integral equation

$$
\xi(t)=K[(q(\mu s)-1) \xi(s)]+\xi_{0}(t):=S[\xi],
$$

where $\xi_{0}(t)$ is defined by (13) and $\left|\xi_{0}(t)\right| \leq m$.

The operator $S$ map the ball $\gamma:\|\xi\| \leq 2 m$ to itself, where $\|\xi\|=\max _{0 \leq t \leq \tilde{\mu}}|\xi(t)|$. We will proof that operator $S$ also is contracting operator. We will consider the operator $S$ on interval $t \in[0, l]$ and $[l, \tilde{\mu}]$. We may take any number greater than 1 for $l$,but we will take $l=1$ for simplicity.

1) Let $t \in(0,1]$, then since $|q(\mu s)-1| \leq \mu s m$, we have

$$
\left|S\left[\xi_{1}\right]-S\left[\xi_{2}\right]\right| \leq h(t)\left\|\xi_{1}-\xi_{2}\right\|,
$$

here $h(t)=\left|\int_{0}^{\tilde{\mu}} K(t, s)(q(\mu s)-1) d s\right|$.

Evaluating $h(t)$ we have got

$$
\begin{aligned}
& h(t) \leq \mu m t \int_{t}^{\tilde{\mu}} \tau^{-2} e^{\tau^{2} / 2} \int_{0}^{\tau} s^{2} e^{s^{2} / 2} d s d \tau \leq \\
& \leq \mu m t \int_{t}^{\tilde{\mu}} \tau^{-1} d \tau \leq \mu m \ln \tilde{\mu}=O(\mu \ln \tilde{\mu}) .
\end{aligned}
$$

2) If $t \in[1, \tilde{\mu}]$ then we will decompose the integral operator $S$ into two Volterra operators:

$$
S[\xi]=\xi_{0}(t)+S_{1}[\xi]+S_{2}[\xi],
$$

where

$$
\begin{aligned}
& S_{1}(\xi)=\int_{0}^{t} X(t) s(q(\mu s)-1) \xi(s) d s- \\
& -t \int_{t}^{\tilde{\mu}} e^{s^{2} / 2}\left[X(s)-s^{-2} e^{-s^{2} / 2}+\right. \\
& \left.+3 s^{-4} e^{-s^{2} / 2}\right](q(\mu s)-1) \xi(s) d s, \\
& S_{2}(\xi)=\mu \int_{t}^{\tilde{\mu}} K_{2}(t, s, \mu) \xi(s) d s, \\
& K_{2}(t, s)=\mu^{-1} t\left[s^{-2}-3 s^{-4}\right](q(\mu s)-1) .
\end{aligned}
$$

Let $\xi_{0}(t)+S_{1}[\xi]=g(\xi)$ and assume $g(\xi)$ as a known function temporary, we will solve the Volterra integral equation

$$
\xi(t)=S_{2}[\xi]+g(\xi)
$$

by the method of successive approximations. The kernel $K_{2}(t, s)=\mu^{-1} t\left[s^{-2}-3 s^{-4}\right](q(\mu s)-1)$ of Volterra operator of $S_{2}[\xi]$ is bounded, since $\left|K_{2}(t, s)\right| \leq m,(1 \leq t \leq \tilde{\mu})$. Therefore 


$$
\xi(t)=g(\xi)+\mu \int_{t}^{\tilde{\mu}} R(t, s, \mu) g(\xi(s)) d s:=T[\xi]
$$

where $R(t, s, \mu)$ is the resolvent of the kernel $K_{2}(t, s)$. For the function $R(t, s, \mu)$ we have got the estimation

$$
|R(t, s, \mu)| \leq m_{1} e^{m \mu(t-s)} .
$$

Since $\mu \int_{t}^{\tilde{\mu}}|R(t, s, \mu)| d s \leq m$, then it is enough to prove that the operator $g(\xi)$ is a contracting one on $t \in[1, \tilde{\mu}]$.We 1 have

$$
\left|g\left(\xi_{1}\right)-g\left(\xi_{2}\right)\right| \leq h_{1}(t)\left|\xi_{1}-\xi_{2}\right|
$$

where

$$
\begin{aligned}
& h_{1}(t)=\int_{0}^{t} X(t) e^{s^{2} / 2} s|q(\mu s)-1| d s+ \\
& +t \int_{t}^{\tilde{\mu}} e^{s^{2} / 2} \mid\left[\left(X(s)-s^{-2} e^{-s^{2} / 2}+\right.\right. \\
& \left.+3 s^{-4} e^{-s^{2} / 2}\right]|q(\mu s)-1| d s
\end{aligned}
$$

Since $|q(\mu s)-1| \leq m \mu s$ and using the property $b)$ of the function $X(t)$ we have got

$$
\begin{aligned}
h_{1}(t) & \leq \mu m X(t) \int_{0}^{t} s^{2} e^{s^{2} / 2} d s+3 \cdot 5 \mu m t \int_{t}^{\tilde{\mu}} s^{-5} d s \leq \\
& \leq \mu m t X(t) \int_{0}^{t} s e^{s^{2} / 2} d s+\frac{3 \cdot 5}{4} \mu m=O(\mu) .
\end{aligned}
$$

It is seen that the operator $S$ is contracting on $[1, \tilde{\mu}]$ with the coefficient of contracting $O(\mu)$. Therefore on the whole interval $[1, \tilde{\mu}]]$ the operator $S[\xi]$ is a contracting operator with the coefficient of contracting $O(\mu \ln \tilde{\mu})$.

Therefore the equation (16) has a unique solution in the ball $\gamma$.

We can write the equation for $\pi_{0}(t)$ in the form

$$
L_{0} \pi_{0}(t)=h\left(t, \pi_{0}\right), \pi_{0}(0)=-y_{0}(0), \pi_{0}(\tilde{\mu})=0,
$$

Here

$$
h\left(t, \pi_{0}\right)=(q(\mu t)-1) \pi_{0}(t)+r[f] \mu t p(\mu t) .
$$

The solution of this problem is represented as

$$
\begin{aligned}
& \pi_{0}(t)=-y_{0}(0) X(t)+\int_{0}^{1 / \mu} K(t, s)\left[(q(\mu t)-1) \pi_{0}(s)+\right. \\
& +r[f] \mu s p(\mu s)] d s .
\end{aligned}
$$

Llemma 5 implies this equation has a unique bounded solution on interval $[0, \tilde{\mu}]$.

Analogously this functions $\pi_{k}(t) \quad(k \in N)$ will be determined from equations (10.к) in unique way and

$$
\left|\pi_{k}(t)\right| \leq m \text {. }
$$

Let

$$
y(x)=\sum_{k=0}^{m}\left(y_{k}(x)+\pi_{k}(t)\right) \mu^{m}+\mu^{m+1} \xi(x, \varepsilon)
$$

The following result is correct

Ttheorem. The solution of the problem(1)-(2)can be represented in the(16) and $|\xi(x, \varepsilon)| \leq m$, that is the series (7) is an asymptotical one for this solution.

\section{The Example. The Comparison of Two Methods: Method of Boundary Layer Functions and Method of Structural Matching}

It is considered the following problem

$$
\begin{aligned}
& \varepsilon y^{\prime \prime}(x)+x y^{\prime}(x)-y(x)=f_{0}+f_{1} x+f_{2} x^{2}, \\
& y(0)=0, y(1)=b
\end{aligned}
$$

a. Method of Boundary Layer Functions

The equation for defining the function $y_{0}(x)$ is

$$
\begin{gathered}
G y_{0}(x):=x y_{0}(x)-y_{0}(x)=f_{0} \\
+f_{2} x^{2}, y_{0}(1)=y^{0}
\end{gathered} .
$$

Hence

$$
y_{0}(x)=-f_{0}+\left(y^{0}+f_{0}-f_{2}\right) x+f_{2} x^{2} .
$$

The equation for the function $\pi_{0}(t)$

$$
\begin{aligned}
L \pi_{0}(t) & :=\pi_{0}^{\prime \prime}(t)+t \pi_{0}^{\prime}(t)-\pi_{0}(t)=f_{1} \mu t, \\
\pi_{0}(0) & =f_{0}, \pi_{0}(\tilde{\mu})=0
\end{aligned}
$$

Then

$$
\begin{aligned}
& \pi_{0}(t)=f_{0} X(t)+f_{1} \mu t \int_{t}^{\tilde{\mu}} \tau^{-2} e^{-\tau^{2} / 2} \int_{0}^{\tau} s^{2} e^{s^{2} / 2} d s d \tau= \\
& =f_{0} X(t)+f_{1} \mu t \ln \mu t+ \\
& +f_{1} \mu t \int_{t}^{\tilde{\mu}} \tau^{-2} e^{-\tau^{2} / 2} \int_{0}^{\tau} e^{s^{2} / 2} d s d \tau \\
& y_{1}(x)=0, \pi_{1}(t)=0 .
\end{aligned}
$$

From the problem $G y_{2}(x)=-2 f_{2}, y_{2}(1)=0$ we have got

$$
y_{2}(x)=2 f_{2}-f_{2} x .
$$

From the equation: $\quad L \pi_{2}(t)=0, \pi_{2}(0)=-2 f_{2}, \pi_{2}(\tilde{\mu})=0$ we obtain

$$
\begin{aligned}
& \pi_{2}(t)=-2 f_{2} X(t)+2 f_{2} t \int_{t}^{\tilde{\mu}} \tau^{-2} e^{-\tau^{2} / 2} \int_{0}^{\tau} s e^{s^{2} / 2} d s d \tau= \\
& =2 f_{2}-2 f_{2} \mu t-4 f_{2} X(t) .
\end{aligned}
$$

For other functions $y_{k}(x)$ we have $y_{k}(x)=0, \pi_{k}(t)=0(k \geq 3)$. Therefore the solution of the problem is 


$$
\begin{aligned}
& y(x)=-f_{0}+\left(y^{0}+f_{0}-f_{2}\right) x+f_{2} x^{2}+ \\
& f_{0} X(x / \mu)+f_{1} x \ln x+ \\
& +f_{1} x \int_{x / \mu}^{\tilde{\mu}} \tau^{-2} e^{-\tau^{2} / 2} \int_{0}^{\tau} e^{s^{2} / 2} d s d \tau+ \\
& +f_{2}(2-x) \mu^{2}+2 f_{2}[1-x-2 X(x / \mu)] \mu^{2} .
\end{aligned}
$$

b. Method of structural matching [11-12]

$b .1)$ The construction of the outer solution.

Definition 1 . The variable $x$ is called the outer variable and it does not depend on the parameter $\varepsilon$.

Definition 2. The solution of the equation of (15) with the initial value condition $y(1)=b$ depending from outer variable $x$ is called the outer solution.

We will seek the outer solution in the form

$$
\begin{aligned}
& y(x)=u_{0}(x)+\varepsilon u_{1}(x)+\varepsilon^{2} u_{2}(x)+ \\
& \varepsilon^{3} u_{3}(x)+\ldots .
\end{aligned}
$$

By inserting (23) to (18) for functions $u_{j}(x)(j=0,1,2 \ldots)$ we the following equations

$$
\begin{aligned}
& M y_{0}(x)=f_{0}+f_{1} x+f_{2} x^{2}, y(1)=b \\
& M y_{1}(x)=-y_{0}{ }^{\prime \prime}(x), \quad y_{1}(1)=0, \\
& M y_{2}(x)=-y_{1} "(x), y_{2}(1)=0, \\
& M y_{3}(x)=-y_{2} "(x), \quad y_{3}(0)=0,
\end{aligned}
$$

Solving these equations we have

$$
\begin{aligned}
& y_{0}(x)=-f_{0}+\left(b+f_{0}-f_{2}\right) x+f_{2} x^{2}+f_{1} x \ln x, \\
& y_{1}(x)=\frac{1}{2} f_{1}\left(x^{-1}-x\right), \\
& y_{2}(x)=-\frac{1}{4} f_{1}\left(x^{-3}-x\right), \\
& y_{3}(x)=\frac{1}{2} f_{1}\left(x^{-5}-x\right), \\
& \cdots \cdots \cdots \cdots \cdots \cdots \cdots \cdots \cdots \cdots \cdots \cdots \cdots \cdots \cdots . . .
\end{aligned}
$$

where $R_{n}$ is a positive rational number. Therefore the outer solution is represented in the form

$$
\begin{aligned}
& u(x)=-f_{0}+\gamma x+f_{2} x^{2}+f_{1} x \ln x+ \\
& \varepsilon \frac{1}{2} f_{1}\left(x^{-1}-x\right)+-\frac{\varepsilon^{2}}{4} f_{1}\left(x^{-3}-x\right)+ \\
& +\frac{\varepsilon^{2}}{2} f_{1}\left(x^{-5}-x\right)+\ldots+ \\
& +\varepsilon^{n}(-1)^{n-1} R_{n} f_{1}\left(x^{-(2 n-1)}-x\right)+\ldots, \\
& \gamma=b+f_{0}-f_{2}
\end{aligned}
$$

or

$$
\begin{aligned}
& u(x)=-f_{0}+\gamma x+f_{2} x^{2}+f_{1} x \ln x- \\
& -\varepsilon \frac{f_{1}}{2} x\left(1-\frac{\varepsilon}{2}+\varepsilon+\ldots+O\left(\varepsilon^{n}\right)\right)+\frac{\varepsilon f_{1}}{2} x^{-1}[1- \\
& \left.-\frac{\varepsilon}{2} x^{-2}+\left(\varepsilon x^{-2}\right)^{2} \ldots+O\left[\left(\varepsilon x^{-2}\right)^{n}\right]+\ldots\right]
\end{aligned}
$$

This series is an asymptotical one on the interval $J=\left[\varepsilon^{\alpha}, 1\right], 0<\alpha<1 / 2$.

b.2) The construction of the inner solution.

We will make the following substitution in (18)

$$
x=\mu t
$$

Then we have got

$$
y^{\prime \prime}(t)+t y^{\prime}(t)-y(t)=f_{0}+f_{1} \mu t+f_{2}(\mu t)^{2} . .
$$

Definition3. The variable $t$ is called the inner variable.

Definition 4. The solution of the equation of (23)with the initial condition $y(0)=0$ depending on the inner variable $t$ is called the inner solution of the problem (18).

Substitution $x=\mu t$ in (18) we obtain

$$
y^{\prime \prime}(t)+t y^{\prime}(t)-y(t)=f_{0}+f_{1} \mu t+f_{2}(\mu t)^{2}, y(0)=0
$$

We can use the second condition $y(1)=y^{0}$ here. Therefore the inner solution is the outer solution of the problem (18).

The solution of this problem is represented in the form

$$
\begin{aligned}
& y(t)=b \mu t-t \int_{t}^{\tilde{\mu}} \tau^{-2} e^{-\tau^{2} / 2} \int_{0}^{\tau} e^{s^{2} / 2}\left(f_{0} s+f_{1} \mu s^{2}+\right. \\
& \left.+f_{2} \mu^{2} s^{3}\right) d s d \tau
\end{aligned}
$$

After integrating by parts we have from this

$$
\begin{aligned}
& f_{0} t \int_{t}^{\tilde{\mu}} \tau^{-2} e^{-\tau^{2} / 2} \int_{0}^{\tau} s e^{s^{2} / 2} d s d \tau=\left.f_{0} t \int_{t}^{\tilde{\mu}} \tau^{-2} e^{-\tau^{2} / 2} e^{s^{2} / 2}\right|_{0} ^{\tau} d \tau= \\
& =f_{0} t \int_{t}^{\tilde{\mu}} \tau^{-2} e^{-\tau^{2} / 2}\left(e^{\tau^{2} / 2}-1\right) d \tau=f_{0} t \int_{t}^{\tilde{\mu}} \tau^{-2} d \tau-f_{0} X(t)= \\
& =f_{0}-f_{0} \mu t-f_{0} X(t), \\
& f_{1} \mu t \int_{t}^{\tilde{\mu}} \tau^{-2} e^{-\tau^{2} / 2} \int_{0}^{\tau} s^{2} e^{s^{2} / 2} d s d \tau= \\
& f_{1} \mu t \int_{t}^{\tilde{\mu}} \tau^{-2} e^{-\tau^{2} / 2}\left(\tau e^{\tau^{2} / 2}-\int_{0}^{\tau} e^{s^{2} / 2} d s\right) d \tau= \\
& =-f_{1} \mu t \ln \mu t- \\
& -f_{1} \mu t \int_{t}^{\tilde{\mu}} \tau^{-2} e^{-\tau^{2} / 2} \int_{0}^{\tau} e^{s^{2} / 2} d s d \tau, \\
& f_{2} \mu t \int_{t}^{\tilde{\mu}} \tau^{-2} e^{-\tau^{2} / 2} \int_{0}^{\tau} s^{3} e^{s^{2} / 2} d s d \tau= \\
& =f_{2} \mu^{2} t\left(\mu^{-1}-t+2 \mu-2 t^{-1}\right)+ \\
& +2 f_{2} \mu^{2} t \int_{t}^{\tilde{\mu}} \tau^{-2} e^{-\tau^{2} / 2} d \tau= \\
& =f_{2} \mu t-f_{2} \mu^{2} t^{2}+2 f_{2} \mu^{3} t-2 f_{2} \mu^{2} \\
& +2 f_{2} \mu^{2} X(t) .
\end{aligned}
$$

Therefore we have got 


$$
\begin{aligned}
& y(t)==-f_{0}+\left(y^{0}+f_{0}-2 f_{2} \mu^{2}\right) X(t)+ \\
& f_{1} \mu t \ln \mu t+\left(f_{0}-f_{2}\right) \mu t+2 f_{2} \mu^{2}+f_{2} \mu^{2} t^{2} \\
& -2 f_{2} \mu^{3} t+f_{1} \mu t \int_{t}^{\tilde{\mu}} \tau^{-2} e^{-\tau^{2} / 2} \int_{0}^{\tau} e^{s^{2} / 2} d s d \tau
\end{aligned}
$$

where $t=x / \mu$. Of course we can receive the expand of the outer solution(22) from (26).

\section{Discussion and Conclusion}

From this example it can be seen, that the method of boundary layer function is at labor-intensive than method structural matching. But the method of structural matching we can apply to construct of asymptotic solution of to almost all singularly perturbed equations and one allow foresee for what small parameter the solution of singular perturbed equation will expand.

\section{Acknowledgement}

I thank Prof. P.Pankov for useful discussion this paper. I also thank the unknown to me the reviewer for his benevolent of my article review.

\section{REFERENCES}

[1] Latta G.E. Singular perturbation problems. California institute of technology, doctoral dissertation, 1951.

[2] Bromberg Nonlinear bending of circular plate under normal pressure, Comm. Pure Appl.Math,Vol.9, 633-659.

[3] Wasow W. Asymptotic Expansions for Ordinary Differential Equations, Dover Publ., Inc., New York, 1987.

[4] Vasil'eva A. B., Butuzov V.F. Asymptotic expansions of solutions of singularly Perturbed equations 3(in Russian), Nauka, Moscow, 1973.

[5] Vasil'eva A. B., Butuzov V.F., Kalachev L. V., The Boundary Function Method for Singular Perturbed Problems, SIAM (Cambridge University Press), 1987.

[6] Vishik,M.L,and Lyusternik,L.A. Regular degeneration and boundary layer for linear differential equations with small parameter(in Russian),Uspehi .Math.Nauk , Vol.12,no1.,3122,1957; Amer.mathSoc.,Transl, Vol.20, 239-364 1962,
[7] M.I. Imanaliev, Asymptotical Methods in the Theory Singularly Perturbed Integro- Differential Systems (in Russian), Ilim, Bishkek, 1972.

[8] V.A. Trenogin, "Development and application of the asymptotical Lusternik-Vishik method", Russian Math. Surveys, Vol.25, No.4,119-156, 1970

[9] Smith D. R., Singular perturbation theory, Cambridge university press, 1985.

[10] O’Malley R. E., Singular Perturbation Methods for Ordinary Differential Equations, Springer-Verlag, 1991.

[11] Dyke Van, Perturbation methods ion fluid mechanics, The parabolic Press, Stanford, 1975.

[12] A. M. Il'in. Matching of Asymptotic Expansions of Solutions of Boundary Value Problems.AMS, 1992.

[13] Lagerstrom P.A. Matched asymptotic expansions. Ideas and techniques.Springer-Verlag, 1988.

[14] Hinch E.J. Perturbation methods. Cambridge university press, 1981

[15] Ali H. Nayfeh. Perturbation Methods, Wiley,2000.

[16] Lindsay A. Skinner Singular Perturbation Theory, Springer-Verlag New York Inc., United States, 2011

[17] K.Alymkulov, A.Z.Zulpukarov, Uniform asymptotic of the boundary problem solution of the singular perturbed equation of the order two with the weak singularity Reports RAN, Vol.398, No.5, 583-586, 2004.

[18] K.Alymkulov, J.K.Jeentaeva, Method of the model Litehill equation structural matching with the regular critical point, Math.Notes, Vol. 79 No.5 643-652,2006.

[19] K.Alymkulov, A.Z.Zulpukarov. On the uniform approximation of the solution of boundary value problem of the singularly perturbed of second order differential equation in the case when unperturbed equation has the regular singular point,Issledovaniepointegro-diffentialuravneniam( $\mathrm{i}$ n Ruissian), Institute math., Nation.Acad.Sc.,Bishkek,Ilim, , v.33, 118-122, 1990.

[20] K.Alymkulov, T.D.Asylbekov, S. F. Dolbeeva Generalization of the boundary function method for solving boundary-value problems for bisingularly perturbed second-order differential equations. Mathem. Notes, , Vol. 94, Issue 3-4, 451-454,2013.

[21] E.F.Mishchenko and N.Kh. Rosov Differential equations with small parameter and relaxation oscillations.,PergamonP ress, New York, 1980. E.f.Mishchenko and N.Kh. Rosov Differential equations with small parameter and relaxation oscillations.,Pergamon Press, New York, 1980. 